\title{
INTRAOPERATIVE EVALUATION OF MYOCARDIAL REVASCULARIZATION DURING MINIMALLY INVASIVE DIRECT CORONARY ARTERY BYPASS BY MEANS OF ULTRASONIC INTEGRATED BACKSCATTER
}

Nobuaki Hirata, MD, Shin Takiuchi, MD, Syouhei Maeda, MD, Shigeaki Ohtake, MD, Yoshiki Sawa, MD, and Hikaru Matsuda, MD, Osaka, Japan

Recently, the concept of minimally invasive surgery has dramatically affected many surgical subspecialties. In the field of coronary artery bypass surgery, minimally invasive direct coronary artery bypass (MIDCAB) is also gaining acceptance. ${ }^{1}$ The indications for MIDCAB are limited, however, and anastomotic complications often occur. ${ }^{2}$ Accordingly, the ability to see real-time changes or the rapid effects of myocardial perfusion in a clinical setting, particularly in the operating room, would be advantageous.

It has been reported that the recovery of cyclic variation of ultrasonic integrated backscatter is a more sensitive reflection of the recovery of regional intramural contractile performance than is a wall motion analysis in areas of acute myocardial infarction. ${ }^{3,4}$ We postulated that the recovery of cyclic variation of ultrasonic integrated backscatter might be a sensitive predictor of the success of myocardial revascularization, even in ischemic, dysfunctional areas. Moreover, the application of this method during MIDCAB could enable real-time intraoperative assessment.

Methods. We studied 6 patients undergoing MIDCAB to the left anterior descending coronary artery. All were men, with a mean age of $57 \pm 6$ years. Three patients had singlevessel disease and the other 3 patients had 2-vessel disease. Their left ventricular function was good (mean left ventricular ejection fraction was $0.70 \pm 0.05$ by ultrasonographic cardiography). The mean operative time was $264 \pm 79$ minutes.

From the First Department of Surgery, Osaka University Medical School, Osaka, Japan.

Received for publication Jan 12, 1999; accepted for publication Jan 20, 1999.

Address for reprints: Nobuaki Hirata, MD, First Department of Surgery, Osaka University Medical School, 2-2, Yamadaoka, Suita, Osaka 565-0871, Japan.

J Thorac Cardiovasc Surg 1999;117:1215-7

Copyright (C) 1999 by Mosby, Inc.

$0022-5223 / 99 \$ 8.00+0 \quad \mathbf{1 2 / 5 4 / 9 7 3 8 1}$
We used a special software package (Acoustic Densitometry) developed by Hewlett-Packard (Hewlett-Packard Company, Palo Alto, Calif) incorporated in a commercially available SONOS 2500 system (Hewlett-Packard). This system is capable of providing either conventional echocardiographic images or 2-dimensional images in which gray levels are displayed proportionally to the integrated backscatter amplitude. Sixty frames from consecutive cardiac cycles (30 frames/s) are displayed after the scan conversion and then stored on an optical disk. When operating in the integrated backscatter imaging mode, the received ultrasonographic signal is amplified, mixed to an appropriate intermediate frequency, phased, and delayed. ${ }^{4}$ All patients underwent a transesophageal echocardiographic examination before and immediately after myocardial revascularization during MID$\mathrm{CAB}$. The short-axis view at the papillary muscle level of the left ventricle was recorded on an optical disk with an electrical sector scanner equipped with acoustic densitometry (carrier frequency $5 \mathrm{MHz}$ ). We depicted 2-dimensional integrated backscatter images, and 60 digital frames ( 2 seconds) were transferred to the optical disc. The transmission power, compression setting, and individual values of the time gain compensation were kept constant throughout the integrated backscatter studies.

Only digitally acquired images can be analyzed with this acoustic densitometry package. Time-intensity waveforms for integrated backscatter were determined in the reconstructed frames. The perfused areas of left anterior descending coronary artery were chosen for the position of the region of interest, and the size of the region of interest was made as large as possible, which excluded endocardial and epicardial reflectors. A single observer adjusted the location of the site on a frame by frame basis to keep it well within the myocardial midwall throughout the cardiac cycle. An ultrasonic integrated backscatter versus time curve was then reconstructed, and we used moderate mode of curve fitting to smooth the time-amplitude waveform. The magnitude of cyclic variation 


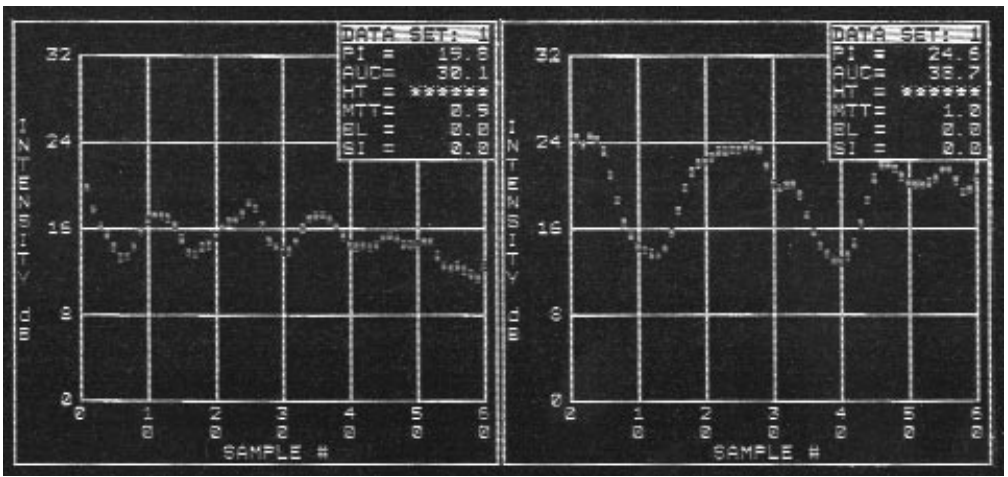

Fig 1. Demonstration of increase in magnitude of cyclic variation of ultrasonographic tissue characterization immediately after revascularization. At this point proximal site of left anterior descending coronary artery was still occluded and myocardial perfusion was obtained only by internal thoracic artery. Left, Before revascularization; Right, immediately after revascularization.

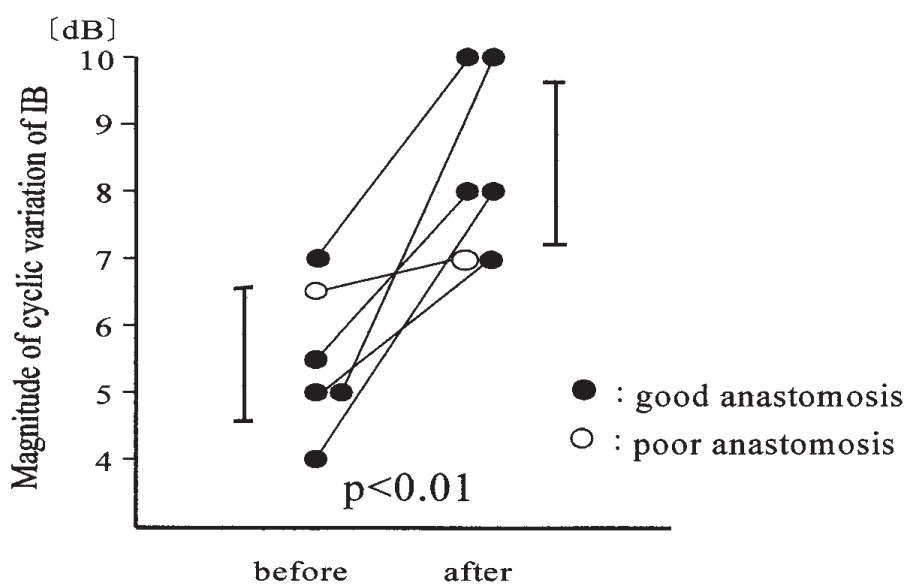

Fig 2. Changes in magnitude of cyclic variation of ultrasonic integrated backscatter $(I B)$ between before and immediately after myocardial revascularization. Magnitude increased in 5 patients with good anastomoses.

of ultrasonic integrated backscatter was determined as the difference between the minimal and maximal values in a cardiac cycle, averaged over at least 2 consecutive beats. Absolute calibration of the imaging systems was not required to measure the cyclic variation of integrated backscattter.

Results. The magnitude of ultrasonic integrated backscatter increased immediately after myocardial revascularization (before $5.5 \pm 1.0 \mathrm{~dB}$ and after $8.8 \pm 0.8 \mathrm{~dB}, P<.01$; Figs 1 and 2 ) in the 5 patients who attained a good anastomosis. This was confirmed by postoperative coronary arteriography. In the case of 1 patient, however, who had an inadequate anastomosis and graft occlusion, the magnitude did not increase (Fig 2).

Discussion. This study revealed that this new method of ultrasonic integrated backscatter enabled an intraoperative evaluation of MIDCAB, in which anastomotic complications can occur, with respect to myocardial tissue characterization. MIDCAB avoids cardiopulmonary bypass and aortic crossclamping. The improvement of regional wall motion in MID-
CAB may occur earlier than seen with the usual coronary artery bypass grafting. Regional wall motion in hibernating myocardium is slow to improve, however, even after percutaneous transluminal angioplasty. Accordingly, this early recovery of cyclic variation of integrated backscatter is consistent with the hypothesis that ultrasonographic tissue characterization provides a useful measure of regional intramural contractile function that is relatively independent of wall motion. The patient who did not show an increase in ultrasonic backscatter had a poor anastomosis.

On the other hand, cyclic variation of integrated backscatter is dependent on the angle between fiber orientation and the ultrasonic beam, known as anisotropy. In the short-axis view at the papillary muscle level of the left ventricle, the anterior or posterior wall is a good object but the lateral wall is not. Data in this study were obtained only from the anteroseptal regions, in which myocardial fiber is oriented nearly perpendicular to the ultrasonic beam, thereby avoiding the anisotropic problems as much as possible. 
Several mechanisms have been postulated to explain the cyclic variation of integrated backscatter: (1) changes in acoustic impedance, which is related to passive elastance, (2) changes in fiber orientation or shape from diastole to systole, and (3) changes in elastic modulus during sarcomere shortening. Wickline and associates ${ }^{5}$ assigned the time-varying acoustic impedance of the intracellular myofibril-sarcomere assembly to 1 of the 2 interfacing zones that are hypothetically responsible for backscatter.

\section{REFERENCES}

1. Calafiore AM, Teodori G, Di Giammarco G, Vitolla G, Iacó A, Iovino T, et al. Minimally invasive coronary artery bypass grafting on a beating heart. Ann Thorac Surg 1997;63(suppl): S72-5.
2. Pagni S, Qaqish NK, Senior DG, Spence PA. Anastomotic complication in minimally invasive coronary bypass grafting. Ann Thorac Surg 1997;63(suppl):S64-7.

3. Milunski MR, Mohr GA, Wear KA, Sobel BE, Miller JG, Wickline SA. Early identification with ultrasonic integrated backscatter of viable but stunned myocardium in dogs. J Am Coll Cardiol 1989;14:462-71.

4. Takiuchi S, Ito H, Iwakura K, Taniyama Y, Nishikawa N, Masuyama $\mathrm{T}$, et al. Ultrasonic tissue characterization predicts myocardial viability in early stage of reperfused acute myocardial infarction. Circulation 1998;97:356-62.

5. Wickline SA, Thomas LJ III, Miller JG, Sobel BE, Pérez JE. The dependence of myocardial ultrasonic integrated backscatter on contractile performance. Circulation 1985;72:183-92. 